\title{
Immunoproteasome-selective inhibitors: the future of autoimmune diseases?
}

\author{
Chong Zhang ${ }^{\ddagger}, 1$, Huajian Zhu ${ }^{\ddagger}, 1$, Jiaan Shao' ${ }^{1}$ Ruoyu $\mathrm{He}^{2}$, Jianjun $\mathrm{Xi}^{2}$, Rangxiao Zhuang*,2 \\ \& Jiankang Zhang $* *, 1$ \\ ${ }^{1}$ School of Medicine, Zhejiang University City College, Hangzhou, 310015, Zhejiang Province, PR China \\ ${ }^{2}$ Department of Pharmaceutical Preparation, Hangzhou Xixi Hospital, Hangzhou, 310023, Zhejiang Province, PR China \\ *Author for correspondence: Tel.: +86 5718648 1960; zhuangrangxiao@sina.com \\ **Author for correspondence: Tel.: +86 5718801 6565; zjk0125@yeah.net \\ $\ddagger$ Authors contributed equally
}

\section{"With persistence in pursuing specific inhibitors targeting immunoproteasomes and promotion of clinical application, this promising target will attract more and more attention."}

First draft submitted: 25 October 2019; Accepted for publication: 31 October 2019; Published online: 27 January 2020

Keywords: autoimmune diseases $\bullet$ design $\bullet$ immunoproteasome $\bullet$ selective inhibitor

The immunoproteasome, a specialized variant of the constitutive one, is predominantly expressed in lymphocytes and monocytes of jawed vertebrates and is considered to be involved in cell-mediated immunity through MHC class-I-mediated antigen presentation [1]. With the illustration of the immunoproteasome structure and functions and following the validation of intimate correlation between immunoproteasome and various diseases, including hematologic malignancies, autoimmune, inflammatory and central nervous system diseases, its importance as a promising drug target has been highlighted [2-5].

\section{Immunoproteasome structure \& selective inhibitors}

Although one drug with two or more targets may bring benefits for therapy, co-inhibition increases the incidence of side effects. As with most anticancer drug targets, it is of great importance for immunoproteasome inhibitors to be developed with appropriate selectivity. Different cellular distributions, expressions and functions of MHC class I antigen processing make the immunoproteasome critical in inhibition of T-cell inflammatory cytokine production and differentiation of $\mathrm{B}$ cells to plasmablasts, while inhibition of constitutive proteasome would bring a more wild range of influences, thus inducing unwanted pharmacological activities and side effects [6].

\section{Structural differences between immuno- \& constitutive proteasomes}

The illustration of immuno- and constitutive proteasome crystal structures has revealed differences in inhibitor specificity for different subunits [2]. For immunoproteasome $\beta 5$ i, configuration alteration at the position of Met 45 as well as mutation of Val31 of constitutive proteasome $\beta 5$ c into Met31 result in a larger $S 1$ pocket. Thus, inhibitors specific for $\beta 5 \mathrm{i}$ require large hydrophobic groups such as Tyr, Trp and Phe at P1. Additionally, when the corresponding residue Ala27 of $\beta 5 \mathrm{c}$ is converted to Ser27, hydrophilic P3 groups become more tolerable to $\beta 5$ i-selective inhibitors. Compared to $\beta 1 \mathrm{c}, S 1$ pocket of $\beta 1 \mathrm{i}$ is more hydrophobic with a smaller size; meanwhile, alternations of sequence reduce and polarize the $S 3$ pocket of $\beta 1$ i subunit. Currently, innovative theories for rational design of $\beta 2$ i-selective inhibitors are not sufficient $[2,7]$.

Actually, potential immunoproteasome inhibitors are required to be not only selective for immunoproteasome over constitutive proteasome, but also display a different inhibitory potency against the three catalytic $\beta$-subunits of immunoproteasome. It is reported that inhibition of cytokine expression requires suppression of at least two active subunits of immunoproteasome simultaneously: $\beta 5 \mathrm{i} / \beta 2 \mathrm{i}$ or $\beta 5 \mathrm{i} / \beta 1 \mathrm{i}[8]$. 


\section{Covalent immunoproteasome-selective inhibitors}

KZR-616, a dual immunoproteasome $\beta 5 \mathrm{i} / \beta 2 \mathrm{i}$ selective inhibitor developed by Kezar Life Sciences, has been recently approved for three Phase II clinical trials for the treatment of several autoimmune diseases [8,9]. Developed based on the optimization of peptidyl epoxyketone derivatives ONX-0914 and PR-924, KZR-616 exhibited potent inhibitory activities toward $\beta 5 \mathrm{i}, \beta 2 \mathrm{i}$ and $\beta 1 \mathrm{i}$ subunits with $\mathrm{IC}_{50}$ values of $0.039,0.131$ and $0.623 \mu \mathrm{M}$, respectively [8]. 18- and 81-fold selectivity against $\beta 5 \mathrm{c}$ and $\beta 1 \mathrm{c}$ and comparative inhibitory activity for two $\beta 2$ subunits were observed.

However, most immunoproteasome-selective inhibitors reported up to now are only able to potently inhibit one of the three catalytic $\beta$-subunits. KZR-504, a dipeptidyl epoxyketone derivative also developed by Kezar Life Sciences, displays forceful inhibitory activity toward $\beta 1 \mathrm{i}$ and moderate activity against $\beta 1 \mathrm{c}$ with $\mathrm{IC}_{50}$ values of 0.05 and $46.27 \mu \mathrm{M}$, respectively $(\beta 1 \mathrm{c} / \beta 1 \mathrm{i}=925)[10] . \mathrm{LU}-001 \mathrm{i}$ is an epoxyketone tetrapeptide containing a proline, which shows a preferential inhibition for $\beta 1 \mathrm{i}\left(\mathrm{IC}_{50}=0.095 \mu \mathrm{M}\right)$ over $\beta 1 \mathrm{c}\left(\mathrm{IC}_{50}=24 \mu \mathrm{M}\right)$ with a 252 -fold selectivity [11]. For $\beta 2 \mathrm{i}$ selective inhibitors, by introduction of a decahydronaphthalene at P1 position, LU-002i was discovered as the most potent one derived from ONX-0914 until now [12]. Besides, by increasing the volume of hydrophobic groups at $\mathrm{P} 1$ position, various $\beta 5$ i selective inhibitors were developed, in which, LU-035i exhibits a 500-fold selectivity [11]. Structure-activity relationship (SAR) studies also evoked the importance of P2 substituents and the chirality of the residue at $\mathrm{P} 3$ position for the potency and selectivity.

While many covalent immunoproteasome-selective inhibitors with electrophilic groups at the C-terminal of the peptide skeleton react with the Thr residue through nucleophilic addition, 2-CA and 4-CA belong to a special class of inhibitors with an $\alpha$-chloroacetamide-containing sidechain, through which a covalent bond is formed with a noncatalytic cysteine of the $\beta 5 \mathrm{i}$ subunit [13]. The cysteine is a strictly conserved nucleophilic residue that is exclusively found in $\beta 5 \mathrm{i}$ subunit and no cysteine residue was located in the $S 4$ pocket of $\beta 5 \mathrm{c}$, thus inducing up to $75-150$-fold selectivity for $\beta 5 \mathrm{i}$ over $\beta 5 \mathrm{c}$ of 2 -CA and 4-CA [2]. Although the inhibitory potency of the two analogs against $\beta 5 \mathrm{i}$ are not optimal ( $\mathrm{IC}_{50}$ values of $6.7 \mu \mathrm{M}$ for 2-CA and $0.6 \mu \mathrm{M}$ for $4-\mathrm{CA}$ ), the distinct covalent binding mechanism presents new opportunities for developing selective immunoproteasome inhibitors.

\section{Noncovalent immunoproteasome-selective inhibitors}

Noncovalent $N, C$-capped peptidomimetics are another option for immunoproteasome inhibitors [14,15]. Insertion of a $\beta$-amino acid into the peptidyl skeleton significantly decreases the inhibitory activities against $\beta 5 \mathrm{c}$, while maintaining potent $\beta 5 \mathrm{i}$ inhibitory activities, thus greatly improving the selectivity [16]. Representative analogs PKS2279 and PKS2252 exhibit 5600- and 13600-fold selectivity for $\beta 5$ i over $\beta 5 c$, which display IC $_{50}$ values of 14 and $5.5 \mathrm{nM}$ against $\beta 5 \mathrm{i}$ and are not active for $\beta 5 \mathrm{c}\left(\mathrm{IC}_{50}\right.$ values more than $\left.70 \mu \mathrm{M}\right)$ [16].

Additionally, nonpeptidyl immunoproteasome-selective inhibitors are well developed in recent years, some of which display favorable immunoproteasome inhibitory activities together with selectivity in comparison with the peptidyl derivatives [17-19]. With more structural variability, optimization of nonpeptidyl analogs would be an important approach for the discovery of novel immunoproteasome-selective inhibitors.

Several constitutive proteasome inhibitors have either been approved for therapy of hematologic malignancies or evaluated in clinical trials and KZR-616, the immunoproteasome-selective inhibitor, is now tested in Phase II clinical trials for various autoimmune diseases, all of which are covalent proteasome inhibitors. Although severe side effects of these drugs are more or less attributed to the covalent interaction, covalent inhibitors still seem to be the most promising drug candidates. Besides, immunoproteasome-selective inhibitors with new covalent binding mechanisms have been reported, which would be an effective complement for discovery of these inhibitors. Nowadays, studies of noncovalent immunoproteasome-selective inhibitors have attracted more and more attention. Reported noncovalent inhibitors exhibited favorable immunoproteasome inhibitory activities and selectivity; however, none of these derivatives have been evaluated in clinical trials and the immanent reasons worth to be further investigated.

\section{Clinical progress}

Although only one immunoproteasome inhibitor is now evaluated in clinical trials, great progress has been achieved for the therapy of autoimmune diseases through inhibition of this promising target. Besides a Phase Ib/II study in patients with systemic lupus erythematosus with and without lupus nephritis (NCT03393013), two other Phase II trials are posted for the evaluation of the safety, tolerability, efficacy, pharmacokinetics and pharmacodynamics of treatment in patients with active polymyositis or dermatomyositis (NCT04033926, 26 July 2019) and active autoimmune hemolytic anemia or immune thrombocytopenia (NCT04039477, 31 July 2019). 
In addition to the above-mentioned autoimmune diseases, the potential of immunoproteasome-selective inhibitors for the treatment of rheumatoid arthritis, Crohn's disease, ulcerative colitis and multiple sclerosis has been validated in preclinical studies. Clinical evaluation of immunoproteasome-selective inhibitors for various autoimmune diseases would be an important milestone for the discovery of this series of drugs.

\section{Future perspective}

For any drug target, corresponding inhibitors with more variety of structural skeletons would guarantee a greater possibility for drug approval. Until now, various peptidyl covalent, peptidyl noncovalent and nonpeptidyl noncovalent immunoproteasome-selective inhibitors have been reported. However, most of these analogs are only able to selectively target one of the three catalytic immunoproteasome subunits, which is not sufficient for inhibition of cytokine expression. Crystal structure elucidation of the immunoproteasome makes it possible for rational design of selective inhibitors for each subunit; however, it is challenging for one inhibitor to fulfill the requirements for targeting two or three different immunoproteasome $\beta$-subunits simultaneously. Following the success of the dual $\beta 5 \mathrm{i} / \beta 2 \mathrm{i}$ selective inhibitor KZR-616, it is believed that more and more selective inhibitors with clinical effects would be developed in the near future.

The current therapy of autoimmune diseases would be changed by immunoproteasome-selective inhibitors. However, no breakthrough has been achieved with other immunoproteasome-related diseases. With persistence in pursuing specific inhibitors targeting immunoproteasomes and promotion of clinical application, this promising target will attract more and more attention.

\section{Financial \& competing interests disclosure}

The authors have no relevant affiliations or financial involvement with any organization or entity with a financial interest in or financial conflict with the subject matter or materials discussed in the manuscript. This includes employment, consultancies, honoraria, stock ownership or options, expert testimony, grants or patents received or pending, or royalties.

No writing assistance was utilized in the production of this manuscript.

\section{References}

1. Murata S, Takahama Y, Kasahara M et al. The immunoproteasome and thymoproteasome: functions, evolution and human disease. Nat. Immunol. 19(9), 923-931 (2018).

2. Huber EM, Basler M, Schwab R et al. Immuno- and constitutive proteasome crystal structures reveal differences in substrate and inhibitor specificity. Cell 148(4), 727-738 (2012).

3. Basler M, Kirk CJ, Groettrup M. The immunoproteasome in antigen processing and other immunological functions. Curr. Opin. Immunol. 25(1), 74-80 (2013).

4. Ettari R, Zappalà M, Grasso S et al. Immunoproteasome-selective and non-selective inhibitors: a promising approach for the treatment of multiple myeloma. Pharmacol. Ther. 182, 176-192 (2018).

5. Miller Z, Ao L, Kim KB et al. Inhibitors of the immunoproteasome: current status and future directions. Curr. Pharm. Des. 19(22), 4140-4151 (2013).

6. Ebstein F, Kloetzel PM, Krüger E et al. Emerging roles of immunoproteasomes beyond MHC class I antigen processing. Cell. Mol. Life Sci. 69(15), 2543-2558 (2012).

7. Lowe J, Stock D, Jap B et al. Crystal structure of the 20 S proteasome from the archaeon T. acidophilum at 3.4 A resolution. Science 268(5210), 533-539 (1995).

8. Johnson HWB, Lowe E, Anderl JL et al. Required immunoproteasome subunit inhibition profile for anti-inflammatory efficacy and clinical candidate KZR-616 ((2S,3R)-N-((S)-3-(cyclopent-1-en-1-yl)-1-((R)-2-methyloxiran-2-yl)-1-oxopropan-2-yl)-3-hydroxy-3-(4methoxyphenyl)-2-((S)-2-(2-morpholinoacetamido)propanamido)propenamide). J. Med. Chem. 61(24), 11127-11143 (2018).

9. Muchamuel T, Anderl J, Fan RA et al. FRI0296 Kzr-616, a selective inhibitor of the immunoproteasome, blocks the disease progression in multiple models of systemic lupus erythematosus (SLE). Ann. Rheum. Dis. 77(S2), 685 (2018).

10. Johnson HWB, Anderl JL, Bradley EK et al. Discovery of highly selective inhibitors of the immunoproteasome low molecular mass polypeptide 2 (LMP2) subunit. ACS Med. Chem. Lett. 8(4), 413-417 (2017).

11. de Bruin G, Huber EM, Xin BT et al. Structure-based design of $\beta 1$ i or $\beta 5$ i specific inhibitors of human immunoproteasomes. J. Med. Chem. 57(14), 6197-6209 (2014).

12. Xin BT, Huber EM, de Bruin G et al. Structure-based design of inhibitors selective for human proteasome $\beta 2 \mathrm{c}$ or $\beta 2 \mathrm{i}$ subunits. J. Med. Chem. 62(3), 1626-1642 (2019). 
13. Dubiella C, Baur R, Cui H et al. Selective inhibition of the immunoproteasome by structure-based targeting of a non-catalytic cysteine. Angew. Chem. Int. Ed. Engl. 54(52), 15888-15891 (2015).

14. Lin G, Chidawanyika T, Tsu C et al. N, C-capped dipeptides with selectivity for mycobacterial proteasome over human proteasomes: role of S3 and S1 binding pockets. J. Am. Chem. Soc. 135(27), 9968-9971 (2013).

15. Blackburn C, Barrett C, Blank JL et al. Optimization of a series of dipeptides with a P3 threonine residue as non-covalent inhibitors of the chymotrypsin-like activity of the human 20S proteasome. Bioorg. Med. Chem. Lett. 20(22), 6581-6586 (2010).

16. Singh PK, Fan H, Jiang X et al. Immunoproteasome $\beta 5$ i-selective dipeptidomimetic inhibitors. ChemMedChem 11(19), 2127-2131 (2016).

17. Cui H, Baur R, Le Chapelain C et al. Structural elucidation of a nonpeptidic inhibitor specific for the human immunoproteasome. ChemBioChem 18(6), 523-526 (2017).

18. Fan H, Angelo NG, Warren JD et al. Oxathiazolones selectively inhibit the human immunoproteasome over the constitutive proteasome. ACS Med. Chem. Lett. 5(4), 405-410 (2014).

19. Sosic I, Gobec M, Brus B et al. Nonpeptidic selective inhibitors of the chymotrypsin-like ( $\beta 5 \mathrm{i}$ ) subunit of the immunoproteasome. Angew. Chem. Int. Ed. Engl. 55(19), 5745-5748 (2016). 